\title{
Optical emulation of photon-pair generation in nonlinear lossy waveguides
}

\author{
Markus Gräfe ${ }^{1,2(a)}$, Diana A. Antonosyan ${ }^{3(a)}$, Alexander S. Solntsev ${ }^{3,4}$, Andrey A. Sukhorukov $^{3}$ \\ and Alexander Szameit ${ }^{1}$ \\ 1 Institut für Physik, Universität Rostock - 18051 Rostock, Germany \\ 2 Institute of Applied Physics, Abbe Center of Photonics, Friedrich-Schiller-Universität Jena \\ Max-Wien-Platz 1, 07743 Jena, Germany \\ 3 Nonlinear Physics Centre, Research School of Physics and Engineering, The Australian National University \\ Canberra, ACT 2601, Australia \\ 4 School of Mathematical and Physical Sciences, University of Technology Sydney - Ultimo, NSW 2007, Australia
}

PACS 42.82.Et - Waveguides, couplers, and arrays

PACS 42.65.Lm - Parametric down conversion and production of entangled photons

PACS $42.25 . \mathrm{Bs}$ - Wave propagation, transmission and absorption

\begin{abstract}
We establish theoretically and demonstrate experimentally that photon-pair generation through spontaneous parametric down-conversion in a nonlinear waveguide with scattering or material losses can be effectively emulated by classical laser light propagation through a specially designed linear waveguide circuit. This platform can represent arbitrary photon and pump losses, with a potential for the emulation of non-Markovian decay. We characterize the photon-pair correlation spectrum and observe its characteristic transformation from the well-known sinc-shape in lossless waveguides towards a Lorentzian shape in the presence of photon loss.
\end{abstract}

Introduction. - Integrated optical circuits enable a stable and scalable realization of quantum logic devices, which can form a basis for the mass production of photonic chips for quantum communication and computation [1-7]. An important challenge is the integration of single-photon sources, which should enable on-chip generation and preparation of quantum states [8-10]. Spontaneous parametric down-conversion (SPDC) in nonlinear waveguides provides an attractive solution for the experimental on-chip generation of correlated and entangled photon pairs [11-15].

Whereas conventional photonic chips are based on dielectric platforms, there is an increasing interest in the development of quantum plasmonic circuits. Such metaldielectric structures are able to strongly confine light to sub-wavelength dimensions, which can enhance the lightmatter interactions. Although metals introduce loss at optical wavelengths, the robust operation of plasmonic circuits with quantum states was recently demonstrated through the observation of quantum interference between single plasmons [16,17]. The realization of spontaneous

(a) These authors contributed equally to this work. frequency conversion in plasmonic structures would provide a route for integrated entangled state generation. However, the effect of losses needs to be carefully considered, since they can nontrivially affect the emerging photon state [18-21].

The establishment of quantum-classical analogies is an active research topic due to the cross-fertilization of ideas [22], with recent examples including simulated quantum walks of entangled photons [23] and the development of classical characterization methods to predict the quantum device performance $[24,25]$. In this work, we suggest and experimentally demonstrate that photon-pair generation through SPDC in a nonlinear lossy waveguide can be emulated in a specially designed linear lossless waveguide lattice. Moreover, the developed waveguide platform can be further applied to optically emulate the effects of non-Markovian (non-exponential) decay and quantum decoherence phenomena [26], which are important for nano-plasmonic circuits [27].

Materials and methods. - For a theoretical description we consider the process of SPDC in a lossy $\chi^{(2)}$ nonlinear waveguide pumped by a quasi-CW laser, where 
a pump photon at frequency $\omega_{\mathrm{p}}$ spontaneously splits into signal and idler photons with corresponding frequencies $\omega_{\mathrm{s}}$ and $\omega_{\mathrm{i}}$, such that $\omega_{\mathrm{p}}=\omega_{\mathrm{s}}+\omega_{\mathrm{i}}$. The effect of linear losses on the SPDC was previously considered in various contexts $[18,19]$ and the detailed theoretical analysis of the emerging photon intensities and correlations in the regime of photon-pair generation was presented in refs. $[20,21]$.

According to the general principles [28], the photon dynamics is governed by the Hamiltonian

$$
\hat{H}=\hat{H}_{\mathrm{nl}}+\hat{H}_{\mathrm{loss}}
$$

where the first part describes the SPDC in a lossless nonlinear medium $\left(\hat{H}_{\mathrm{nl}}\right)$ and the latter one represents the linear losses, which model the coupling to an external reservoir $\left(\hat{H}_{\text {loss }}\right)$.

The SPDC process in the absence of losses and in the undepleted classical pump approximation is governed by the Hamiltonian [29] $\hat{H}_{\mathrm{nl}}(z)=\beta_{\mathrm{s}}^{(0)} \hat{a}_{\mathrm{s}}^{\dagger} \hat{a}_{\mathrm{s}}+$ $\beta_{\mathrm{i}}^{(0)} \hat{a}_{\mathrm{i}}^{\dagger} \hat{a}_{\mathrm{i}}+\left[A(z) \chi(z) \hat{a}_{\mathrm{s}}^{\dagger} \hat{a}_{\mathrm{i}}^{\dagger}+A^{*}(z) \chi^{*}(z) \hat{a}_{\mathrm{s}} \hat{a}_{\mathrm{i}}\right]$, where $\hat{a}_{\mathrm{s}, \mathrm{i}}^{\dagger}$ and $\hat{a}_{\mathrm{s}, \mathrm{i}}$ are the creation and annihilation operators for the signal (s) and idler (i) photons with the commutators $\left[\hat{a}_{\mathrm{s}}, \hat{a}_{\mathrm{s}}^{\dagger}\right]=\left[\hat{a}_{\mathrm{i}}, \hat{a}_{\mathrm{i}}^{\dagger}\right]=1, \beta_{\mathrm{s}, \mathrm{i}}^{(0)}$ are the signal and idler propagation constants relative to the pump (we consider a narrowband regime with $\beta_{\mathrm{s}, \mathrm{i}}^{(0)}$ not depending on wavelength), $A$ is the pump amplitude, and $\chi$ is the effective quadratic nonlinearity coefficient in the waveguide.

We first analyze the effect of material or scattering losses which are Markovian, i.e., there is no correlation between the loss processes at different positions along the waveguide. Under the condition of negligible thermal fluctuations, the Hamiltonian governing the losses can be written as $\hat{H}_{\text {loss }}(z)=\sqrt{2 \gamma_{\mathrm{i}}}\left[\hat{a}_{\mathrm{i}} \hat{b}_{\mathrm{i}}^{\dagger}(z)+\hat{a}_{\mathrm{i}}^{\dagger} \hat{b}_{\mathrm{i}}(z)\right]+$ $\sqrt{2 \gamma_{\mathrm{s}}}\left[\hat{a}_{\mathrm{s}} \hat{b}_{\mathrm{s}}^{\dagger}(z)+\hat{a}_{\mathrm{s}}^{\dagger} \hat{b}_{\mathrm{s}}(z)\right]$, Here, the $\gamma_{\mathrm{s}, \mathrm{i}}$ are the linear loss coefficients and the operators $\hat{b}_{\mathrm{s}, \mathrm{i}}^{\dagger}(z)$ describe photon modes of an external reservoir which are occupied by signal and idler photons that are lost from the waveguide at coordinate $z$. Such a description of losses is a standard approach which has been used, for example, to describe lossy plasmonic waveguides [30]. The commutator relation conforms to Markovian statistics, $\left[\hat{b}_{\mathrm{i}}\left(z_{1}\right), \hat{b}_{\mathrm{i}}^{\dagger}\left(z_{2}\right)\right]=\left[\hat{b}_{\mathrm{s}}\left(z_{1}\right), \hat{b}_{\mathrm{s}}^{\dagger}\left(z_{2}\right)\right]=$ $\delta\left(z_{1}-z_{2}\right)$.

We analyze the photon-pair generation while neglecting multi-photon-pair processes for the appropriately attenuated pump power. Due to losses, one or both photons from a pair can be lost. Nevertheless, the two-photon state can be distinguished from the single-photon or vacuum state by performing correlation measurements with two singlephoton detectors, as schematically illustrated in fig. 1(a). The two-photon wave function is governed by the following equation [20]:

$$
\mathrm{i} \frac{\mathrm{d} \Phi(z)}{\mathrm{d} z}-\left(\Delta \beta^{(0)}-\mathrm{i} \gamma\right) \Phi(z)-A(z) \chi(z)=0,
$$

with the initial condition $\Phi(z=0)=0$. Here, $\Delta \beta^{(0)}=$ $\beta_{\mathrm{s}}^{(0)}+\beta_{\mathrm{i}}^{(0)}$ is the phase mismatch and $\gamma=\gamma_{\mathrm{s}}+\gamma_{\mathrm{i}}$ is

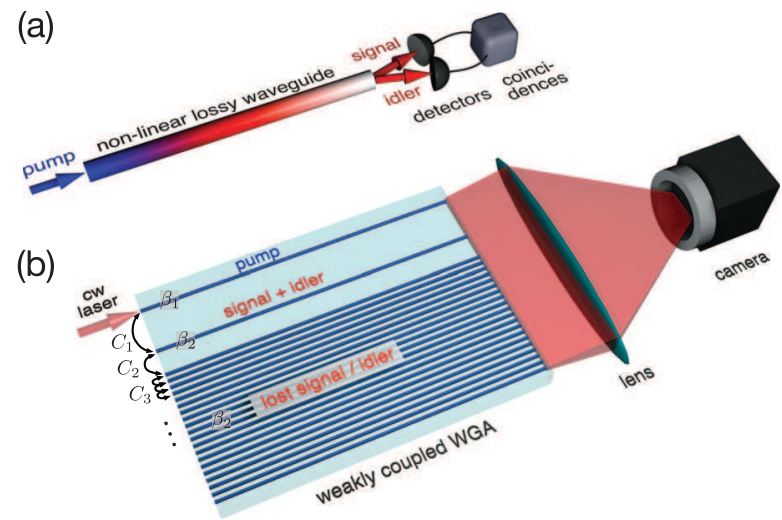

Fig. 1: (Colour online) (a) Scheme of photon-pair generation through SPDC in a nonlinear waveguide with loss. The photon states are defined by frequency-dependent phase mismatch and losses. The probability of generation is defined by correlations of signal and idler counts at single-photon detectors. (b) Sketch of the experimental setup with the classical optical emulator circuit based on coupled linear waveguides. Laser light is launched into the the first waveguide and the output intensities are imaged on a CCD camera.

the combined photon loss coefficient. Importantly, the biphoton evolution is sensitive to the combined signal and idler losses, rather then individual loss coefficients. Taking into account the pump loss rate $\gamma_{\mathrm{p}}$ such that $A(z)=A(z=0) \mathrm{e}^{-\gamma_{\mathrm{p}} z}$, the probability to simultaneously detect signal and idler photons by two detectors is found to be

$$
I(z)=|\Phi(z)|^{2}=\frac{2 A^{2} e^{-\tilde{\gamma}_{+} z}\left\{\cosh \left(\tilde{\gamma}_{-} z\right)-\cos \left(\Delta \beta^{(0)} z\right)\right\}}{\left(\Delta \beta^{(0)}\right)^{2}+\left(\tilde{\gamma}_{-}\right)^{2}},
$$

where $\tilde{\gamma}_{ \pm}=\gamma \pm \gamma_{\mathrm{p}}$.

We now present an approach to emulate the photonpair generation in a nonlinear lossy waveguide by means of a classical lossless circuit of coupled optical waveguides. In the optical emulator, a classical complex optical mode amplitude evolution along a waveguide directly matches the complex biphoton wave function dynamics. We initially consider the case of a lossless pump propagation with $\gamma_{\mathrm{p}}=0$, and accordingly, $A(z)=A(0)$.

The emulating circuit should represent the biphoton wave fuction evolution according to the Hamiltonian that contains the nonlinear and loss terms shown in eq. (1). Interestingly, the nonlinear part describing the biphoton generation by an undepleted pump in a lossless waveguide can be emulated by directionally coupled waveguides [23]. Furthermore, Markovian losses can be emulated through the coupling between one waveguide and a semi-infinite waveguide array [26]. In our circuit we combine both approaches, hence, the resulting design is depicted in fig. 1(b).

Laser light is launched into the first waveguide representing the pump. The weak coupling of the first to the 
second waveguide $\left(C_{1}\right)$ emulates the generation of biphotons. Accordingly, the light amplitude in the second waveguide emulates the biphoton wave function. Additionally, the propagation constant in the first waveguide is detuned with respect to the other one's in order to account for the phase mismatch $\Delta \beta^{(0)}$. The photon losses are emulated by light coupling out of the second to the third waveguide $\left(C_{2}\right)$, followed by the propagation through an array of waveguides (waveguide number $n \geq 3$ ) with high coupling rate $C_{3}>C_{2}>C_{1}$. We consider the array to be semi-infinite, or in practice, sufficiently wide to avoid reflections from the edge.

We now prove the mathematical correspondence between the biphoton wave function evolution and the light propagation in the linear waveguide circuit. The laser light propagation in the circuit is described by the coupledmode equations [31]

$$
\mathrm{i} \frac{\mathrm{d} \psi_{n}}{\mathrm{~d} z}-\beta_{n} \psi_{n}+C_{n-1} \psi_{n-1}+C_{n} \psi_{n+1}=0,
$$

where $n \geq 1$ are the waveguide numbers, $\psi_{n}$ are the complex classical mode amplitudes, $C_{n}$ are the coupling coefficients proportional to the mode overlaps in the neighboring waveguides, and $\beta_{n}$ are the waveguide propagation constants. We set $C_{0} \equiv 0$, and in the loss-emulating section $C_{n>4}=C_{3}$ as well as $\beta_{n>3}=\beta_{2}$ (see fig. 1). By applying the Fourier transform $\psi_{n}(z)=\int \mathrm{e}^{i \beta z} f_{n}(\beta) \mathrm{d} \beta$ to eqs. (4) we obtain the corresponding coupled algebraic equations

$$
-\beta f_{n}+C_{n-1} f_{n-1}+C_{n} f_{n+1}-\beta_{n} f_{n}=0 .
$$

The solution of eqs. (5) in the semi-infinite section with the homogeneous coupling $C_{3}$ for $n \geq 3$ has the form of a discrete Bloch wave [31], $f_{n}=f_{3} \exp [i k(n-3)]$. The wave number $k$ satisfies the spatial dispersion relation $-\beta+2 C_{3} \cos (k)-\beta_{2}=0$, following from eqs. (5). Next we take into account that for $n>3$ the coupling between the loss-emulating waveguides is much larger than the propagation constant, $C_{3} \gg\left|\beta, \beta_{2}\right|$, which means that $\cos (k) \ll$ 1 , and thus, $k=\kappa+\pi / 2$, with $|\kappa| \ll 1$ since the array is semi-infinite with no back reflections. Therefore, we can rewrite the dispersion relation as $\beta=-\beta_{2}-2 C_{3} \sin (\kappa) \approx$ $-\beta_{2}-2 C_{3} \kappa$. Employing this dispersion, we find from eq. (5) a relation between the modes in the second and the third waveguides $f_{3}=\left(C_{2} / C_{3}\right) \exp (i k) f_{2} \approx\left(C_{2} / C_{3}\right) i f_{2}$, and accordingly in real space $\psi_{3}(z) \approx\left(c_{2} / c_{3}\right) i \psi_{2}(z)$. We substitute this expression in eqs. (4) for $n=2$ and obtain

$$
\mathrm{i} \frac{\mathrm{d} \psi_{2}}{\mathrm{~d} z}-\left(\beta_{2}-\mathrm{i} \frac{C_{2}^{2}}{C_{3}}\right) \psi_{2}+C_{1} \psi_{1}=0 .
$$

Further, we consider the undepleted pump regime with $C_{1} z \ll 1$, and in this case $\psi_{1}(z) \approx \psi_{1}(0) \exp \left(i \beta_{1} z\right)$.

Finally, comparing eq. (2) for the biphoton state and eq. (6) for the classical light evolution in the second waveguide unambiguously shows their equivalence. We require that $\psi_{2}(z) \equiv \Phi(z)$ and obtain the following relations:

$$
\gamma=\frac{C_{2}^{2}}{C_{3}}, \quad \psi_{1}(0)=-\frac{A \chi}{C_{1}}, \quad \beta_{1}=0, \quad \beta_{2}=\Delta \beta^{(0)} .
$$

In this vein, the considered waveguide lattice shown in fig. 1(b) effectively emulates the SPDC in a nonlinear lossy waveguide. Specifically, the intensity in waveguide $n=2$ represents the probability of the signal-idler biphoton generation at the output. In order to perform an experimental analysis we fabricated waveguide structures using the femtosecond laser direct writing technique [1,32]. In doing so, the propagation constant detuning of the pump guide was controlled via the inscription velocity. The length of the waveguides is $7.6 \mathrm{~cm}$, and their transverse separation determines the coupling coefficient between them [33]. Their detuning emulating the phase mismatch is controlled via the inscription velocity. While the structures emulate different mismatch and loss regimes, the coupling between the first and the second waveguide stays constant at $C_{1}=0.0575 \mathrm{~cm}^{-1}$. Thus, the effective pump amplitude $A$ is kept constant for all experiments at the same laser power $\left(\left|\psi_{1}\right|^{2}\right)$ according to eq. (7). For the classical-optical emulation a laser light of $633 \mathrm{~nm}$ was injected into the first waveguide and the output intensity of the complete structure was observed via a CCD camera, as illustrated in fig. 1(b).

Results and discussion. - As a first step we analyze the simplest case without loss $(\gamma=0)$. Then the intensity for the signal-idler biphoton provided by eq. (3) exhibits a characteristic sinc-shape,

$$
I(z)=A^{2} z^{2} \operatorname{sinc}^{2}\left(\frac{\Delta \beta^{(0)} z}{2}\right) .
$$

Figure 2(a) shows this biphoton intensity evolution along a nonlinear waveguide for different phase mismatches.

Along theses lines the emulation of SPDC in a nonlinear lossless waveguide is realized by just two linear coupled waveguides $(n=1,2)$. In accordance with eq. (7) $C_{2}=0$ represents the lossless case, such that there is no coupling to the loss-emulating array with $n \geq 3$. The corresponding experimental results are presented in fig. 2(b) as blue circles. For comparison we plotted the quantum solution with a solid red line and the numerical solution of the coupled-mode equations representing the emulating circuit with a dashed green line. In the theoretical simulations the pump amplitude is normalized according to the experimental results and is constant for the lossless and the loss-emulating case at $A \chi=0.6238$. The data shows excellent agreement between both theoretical curves and the experimental measurements across a wide range of phase mismatches.

Next, we investigate the SPDC in a nonlinear lossy waveguide and its classical emulation. In the presence of loss the biphoton intensity dependence on the phase mismatch transforms from a sinc-shape to a broadened 

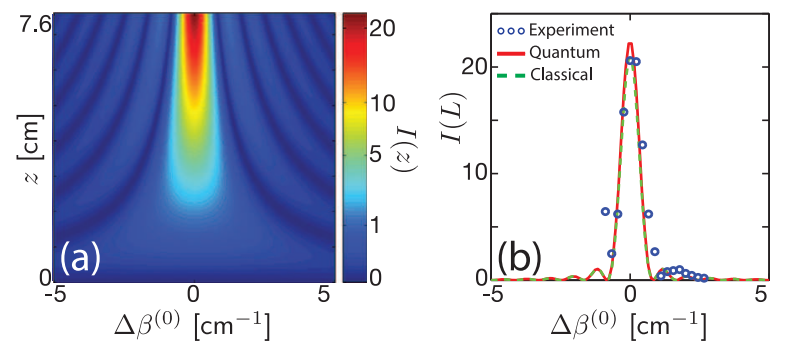

Fig. 2: (Colour online) Emulation of photon-pair generation in a lossless waveguide $(\gamma=0)$. (a) Quantum solution for biphoton intensity evolution in a nonlinear waveguide according to eq. (8). (b) Output intensity vs. the phase mismatch: experimental measurements (blue circles), quantum solution by eq. (8) (solid red line), and numerical solution of the emulating linear coupled-mode eqs. (4) (dashed green line). The length of the waveguides is $L=7.6 \mathrm{~cm}$, the coupling coefficient between the first and second waveguides is $C_{1}=0.0575 \mathrm{~cm}^{-1}$, and $C_{2}=0$. Fabrication deviations of the laser written waveguides result in an uncertainty of the phase mismatch (difference in propagation constants) but is within the marker size.
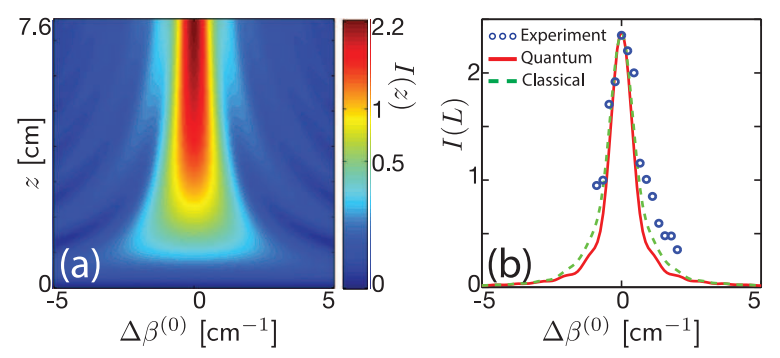

Fig. 3: (Colour online) Emulation of photon-pair generation in a waveguide with loss $\left(\gamma=0.397 \mathrm{~cm}^{-1}\right)$. The corresponding experimental couplings are $C_{2}=0.63 \mathrm{~cm}^{-1}$ and $C_{3}=1.0 \mathrm{~cm}^{-1}$. The quantum solution is given by eq. (3). Other parameters and notations are the same as in fig. 2 .

Lorentzian shape $[19,20]$, as described by eq. (3), which is imaged in fig. 3(a). This phenomenon can be intuitively explained as follows. When the process of SPDC is phasemismatched, the pump generates the photon pairs with an opposite phase after propagating a coherence length, resulting in destructive interference. However this process is interrupted if either signal or idler photon is lost, since such photons do not interfere with photon-pairs [34-36]. This phenomenon effectively broadens the phase-matching curve for higher losses and smoothes out the minima present in the sinc shape corresponding to the lossless case.

The emulating system now includes the loss-emulating array with waveguide couplings that determine the loss coefficient (see eq. (7)). The loss-emulating array consists of 20 waveguides $(n=3, \ldots, 22)$, which is sufficient to avoid reflections from the boundary. As a result, we point out the very strong agreement of the quantum calculation compared with the coupled-mode analysis. Strictly speaking, our realized phase mismatches partially exceed the limit $C_{3} \gg\left|\beta, \beta_{2}\right|$ of our theoretical derivations. Nevertheless, the almost perfect overlap of both theory curves

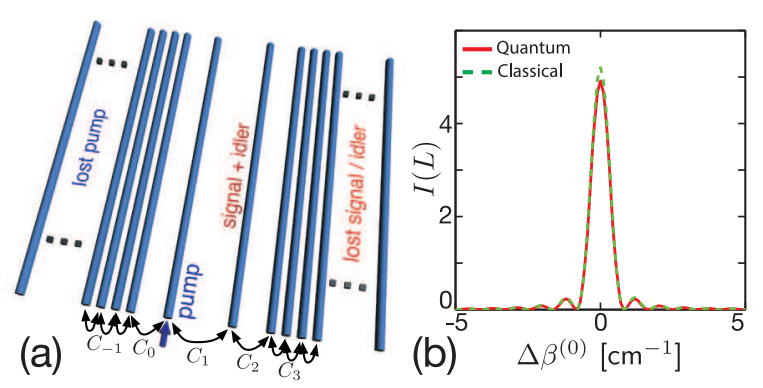

Fig. 4: (Colour online) Emulation of photon-pair generation in a waveguide with photon and pump losses. (a) Design of the corresponding linear circuit with an additional array at $n \leq 0$ emulating the pump loss. (b) Output intensity vs. the phase mismatch: quantum solution by eq. (3) (solid red line) and numerical solution of the emulating linear system by eqs. (4) (dashed green line). The pump and photon losses are matched, $\gamma_{\mathrm{p}}=\gamma=0.1 \mathrm{~cm}^{-1}, C_{0}=C_{2}=0.3162 \mathrm{~cm}^{-1}$, and $C_{-1}=C_{3}=1 \mathrm{~cm}^{-1}$. Other parameters are the same as in fig. 3.
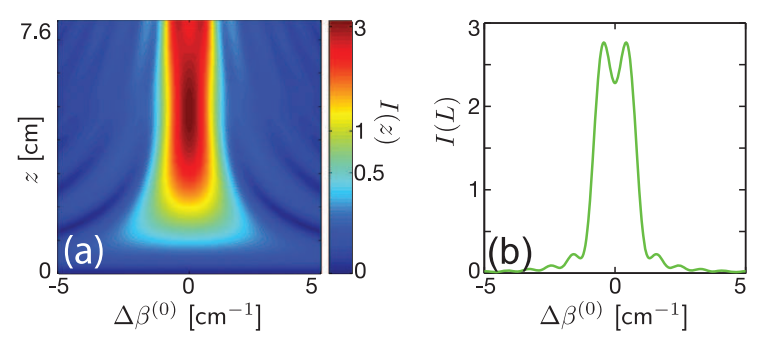

Fig. 5: (Colour online) Emulation of photon-pair generation in a waveguide with non-Markovian loss. The corresponding experimental couplings are $C_{2}=0.37 \mathrm{~cm}^{-1}$ and $C_{3}=0.35 \mathrm{~cm}^{-1}$. Other parameters and notations are the same as in fig. 2. Note the formation of the dip at $\Delta \beta^{(0)}=0$.

show that our predictions are still valid. This is even more confirmed by the very good agreement of the experimental data with both theoretical curves.

Additionally, we point out that the circuit can be designed to emulate the effect of pump losses as well. This can be achieved by placing a second loss-emulating array on the left of the pump waveguide, as illustrated in fig. 4(a). The corresponding coupling coefficients are found analogously to eq. (7) as $\gamma_{\mathrm{p}}=C_{0}^{2} / C_{-1}$. We also set $C_{n \leq-2}=C_{-1}$ and $\beta_{n \leq 0}=0$. Interestingly, when the pump loss is equal to the combined signal and idler losses, such that $\gamma_{\mathrm{p}}=\gamma$ then, the biphoton dependence on the phase mismatch recovers a sinc-shape, just like in the lossless case, which is depicted in fig. 4(b).

Finally, we note that by altering the arrangement of the waveguides, the developed platform can be tailored to emulate the effects of non-Markovian (non-exponential) decay and quantum decoherence phenomena [26]. This can be achieved by breaking the condition $C_{2} \ll C_{3}$. A particularly interesting situation arises when $C_{2} \approx C_{3}$. In this case we observe the suppression of the SPDC efficiency in the regime of exact phase matching, when $\Delta \beta^{(0)}=0$. This effect gradually develops along the propagation direction, 
as shown in fig. 5(a). The output profile presented in fig. 5(b) features a clearly visible dip in the SPDC intensity spectrum, which would not be possible in the case of conventional Markovian losses. Essentially, non-Markovian losses enable revivals and fractional decoherence of lost photons [26] enabling back-and-forth dynamics between the photon pair and the loss reservoir, which qualitatively may behave similaly to the phase-mismatch facilitating a back-and-forth between the photon pair and the pump. The simulation of non-Markovian losses is important, because it may help to study potentially attractive systems such as a photonic crystal cavity coupled to a waveguide [37].

Conclusions. - In conclusion, we presented the theoretical concept and the experimental realization of a classical optical emulator of quantum photon-pair generation in a lossy quadratic nonlinear waveguide. Our system fully emulates the spatial evolution of two-photon states and corresponding time-averaged correlations within the framework of the considered approximations (undepleted pump, single-guided mode SPDC, and no higher-order photon pairs), even though the temporal statistics and quantum fluctuations are not captured. The transformation of a conventional sinc-shaped photon-pair correlation spectrum into a Lorenzian shape in the presence of signal and idler photon losses was demonstrated. Our approach based on a linear waveguide lattice is very flexible, and can further enable emulations of non-Markovian processes and pump losses, which are a common feature in photonic nanostructures including plasmonic circuits.

This work has been supported by the Australian Research Council (Discovery Projects DP130100135 and DP160100619), the Erasmus Mundus NANOPHI Project (contract number 2013 5669/002-001), the German Ministry for Education and Research (Alexander von Humboldt Fellowship), the Deutsche Forschungsgemeinschaft (grants SZ276/7-1, SZ276/9-1, SZ276/12-1, SZ276/15-1, BL574/13-1), and the Australia-Germany Joint Research Cooperation Scheme (grant no. 57216238).

\section{REFERENCES}

[1] Meany T., Gräfe M., Heilmann R., Prez-Leija A., Gross S., Steel M. J., Withford M. J. and Szameit A., Laser Photon. Rev., 9 (2015) 363.

[2] Gräfe M., Heilmann R., Lebugle M., Guzman-Silva D., Perez-Leija A. and Szameit A., J. Opt., 18 (2016) 103002.

[3] Politi A., Cryan M. J., Rarity J. G., Yu S. Y. and O'Brien J. L., Science, 320 (2008) 646.

[4] Metcalf B. J., Spring J. B., Humphreys P. C., Thomas-Peter N., Barbieri M., Kolthammer W. S., Jin X. M., Langford N. K., Kundys D., Gates J. C., Sмith B. J., Sмith P. G. R. and Walmsley I. A., Nat. Photon., 8 (2014) 770.
[5] Gräfe M., Heilmann R., Perez-Leija A., Keil R., Dreisow F., Heinrich M., Moya-Cessa H., Nolte S., Christodoulides D. N. and Szameit A., Nat. Photon., 8 (2014) 791.

[6] Najafi F., Mower J., Harris N. C., Bellei F., Dane A., Lee C., Hu X. L., Kharel P., Marsili F., Assefa S., Bergaren K. K. and Englund D., Nat. Commun., 6 (2015) 5873.

[7] Crespi A., Ramponi R., Osellame R., Sansoni L., Bongioanni I., Sciarrino F., Vallone G. and Mataloni P., Nat. Commun., 2 (2011) 566.

[8] Collins M. J., Xiong C., Rey I. H., Vo T. D., He J., Shahnia S., Reardon C., Krauss T. F., Steel M. J., Clark A. S. and Eggleton B. J., Nat. Commun., 4 (2013) 2582.

[9] Silverstone J. W., Bonneau D., Ohira K., Suzuki N., Yoshida H., Iizuka N., Ezaki M., Natarajan C. M., Tanner M. G., Hadfield R. H., Zwiller V., Marshall G. D., Rarity J. G., O'Brien J. L. and Thompson M. G., Nat. Photon., 8 (2014) 104.

[10] Harris N. C., Grassani D., Simbula A., Pant M., Galli M., Baehr-Jones T., Hochberg M., Englund D., Bajoni D. and Galland C., Phys. Rev. X, 4 (2014) 041047.

[11] Zhang Q., Xie X. P., Takesue H., Nam S. W., Langrock C., Fejer M. M. and Yamamoto Y., Opt. Express, 15 (2007) 10288.

[12] Yu X. Q., Xu P., Xie Z. D., Wang J. F., Leng H. Y., Zhao J. S., Zhu S. N. and Ming N. B., Phys. Rev. Lett., 101 (2008) 233601.

[13] Krapick S., Herrmann H., Quiring V., Brecht B., Suche H. and Silberhorn C., New J. Phys., 15 (2013) 033010.

[14] Solntsev A. S., Setzpfandt F., Clark A. S., Wu C. W., Collins M. J., Xiong C., Schreiber A., Katzschmann F., Eilenberger F., Schiek R., Sohler W., Mitchell A., Silberhorn C., Eggleton B. J., Pertsch T., Sukhorukov A. A., Neshev D. N. and Kivshar Y. S., Phys. Rev. X, 4 (2014) 031007.

[15] Jin H., Liu F. M., Xu P., Xia J. L., Zhong M. L., Yuan Y., Zhou J. W., Gong Y. X., Wang W. and Zhu S. N., Phys. Rev. Lett., 113 (2014) 103601.

[16] Heeres R. W., Kouwenhoven L. P. and Zwiller V., Nat. Nanotechnol., 8 (2013) 719.

[17] Fakonas J. S., Lee H., Kelaita Y. A. and Atwater H. A., Nat. Photon., 8 (2014) 317.

[18] Caves C. M. and Crouch D. D., J. Opt. Soc. Am. B, 4 (1987) 1535.

[19] Klyshko D., Photons and Nonlinear Optics (Gordon and Breach, New York) 1988.

[20] Antonosyan D. A., Solntsev A. S. and Sukhorukov A. A., Phys. Rev. A, 90 (2014) 043845.

[21] Helt L. G., Steel M. J. and Sipe J. E., New J. Phys., 17 (2015) 013055.

[22] Longhi S., Laser Photon. Rev., 3 (2009) 243.

[23] Gräfe M., Solntsev A. S., Keil R., Sukhorukov A. A., Heinrich M., Tünnermann A., Nolte S., Szameit A. and Kivshar Y. S., Sci. Rep., 2 (2012) 562.

[24] Liscidini M. and Sipe J. E., Phys. Rev. Lett., 111 (2013) 193602.

[25] Helt L. G. and Steel M. J., Opt. Lett., 40 (2015) 1460. 
[26] Longhi S., Opt. Lett., 38 (2013) 4884.

[27] Ginzburg P. and Zayats A. V., Opt. Express, 20 (2012) 6720 .

[28] Lloyd S., Science, 273 (1996) 1073.

[29] Mandel L. and Wolf E., Optical Coherence and Quantum Optics (Cambridge University Press, New York) 1995.

[30] Tame M. S., Lee C., Lee J., Ballester D., Paternostro M., Zayats A. V. and Kim M. S., Phys. Rev. Lett., 101 (2008) 190504.

[31] Lederer F., Stegeman G. I., Christodoulides D. N., Assanto G., Segev M. and Silberberg Y., Phys. Rep., 463 (2008) 1.
[32] Davis K. M., Miura K., Sugimoto N. and Hirao K., Opt. Lett., 21 (1996) 1729.

[33] Szameit A., Dreisow F., Pertsch T., Nolte S. and Tunnermann A., Opt. Express, 15 (2007) 1579.

[34] Zou X. Y., Wang L. J. and Mandel L., Phys. Rev. Lett., 67 (1991) 318.

[35] Lemos G. B., Borish V., Cole G. D., Ramelow S., Lapkiewicz R. and Zeilinger A., Nature, 512 (2014) 409.

[36] Kalashnikov D. A., Paterova A. V., Kulik S. P. and Krivitsky L. A., Nat. Photon., 10 (2016) 98.

[37] Lei C. U. and Zhang W.-M., Phys. Rev. A, 84 (2011) 052116 . 